\title{
The method of array antenna constructive synthesis on the basis of neural network approach
}

\author{
Sergey Mishchenko ${ }^{*}$, Vitaliy Shatskiy, Alexey Litvinov, and Denis Eliseev \\ FSPC "Rostov research institute of radiocommunications", 344038, Rostov-on-Don, Russia
}

\begin{abstract}
The method to decision constructive synthesis of array antennas was conducted. The method usefull when antenna elements can be in discreste states (for example: active element, passive element, excluded item, active element with discrete nominal of output power e.t.c). The method is based on neural network approach. The structure of a neural network consist of a classifying neural network and several approximating neural networks is substantiated. Input signals correspond to phase centers of array antenna elements. Number of output signals in classifying part is equal to discrete status of antenna element. Each approximating part of network has one output signal wich correspond to continious meaning. Separate parts of network preliminary learning with error back propagation method. The genetic algorithm of neural network learning with limited number of training coefficients is proposed. Examples of solving problems of constructive synthesis, with different indicators of the quality of neural network training are given.
\end{abstract}

\section{Introduction}

In the theory of antenna synthesis the problem of solving the amplitude-phase distribution (APD) is usually considered at a given antenna geometry, the requirements for the characteristics of radiation pattern and restrictions on the range of acceptable solutions. However, in practice, the problems of constructive synthesis are often associated with the definition of optimal APD and the configuration of the antenna in the sense of the chosen criterion. The decision of such problems is too difficult. So in known literature usually use some simplifications. In [1] authors consider the constructive synthesis of linear nonequidistant array antenna (AA) with known APD. In [2] authors choose optimal APD for predefined configurations of AA. In [3] linear array antenna contains a small number of elements. However on practice it is need to decide the problems of constructive synthesis of array antenna with account of mutual influence elements, discrete and continuous states of control and active devices of array antenna. In this case array antenna can include active elements, passive elements and it is need to exclude some elements from array. In the conference [4] we suggested the universal neural network (NN) approach to decision such problems. The structure of a $\mathrm{NN}$ consist of a classifying neural network and several approximating neural networks is substantiated. Input signals correspond to phase centers

*Corresponding author: mihome@yandex.ru 
of AA elements. Number of output signals in classifying part is equal to discrete status of antenna element. Each approximating part of network has one output signal wich correspond to continious meaning. Separate parts of network preliminary learning with error back propagation method. The genetic algorithm of neural network learning with limited number of training coefficients is proposed.

The purpose of the work is to create a method of constructive synthesis of array antennas with account of mutual influence of antenna elements on the basis of neural network approach.

\section{The foundation for the syntesis method}

All antenna elements influence to each one. It influence characterizes normed resistance matrix $\mathbf{Z}$. With account matrix $\mathbf{Z}$ the radiation pattern of AA can be calculated with formula

$$
F(u, v)=\mathbf{A}^{T} \mathbf{Z f}(u, v)
$$

where

$$
\mathbf{f}(u, v)=\left(f_{0}(u, v) \exp \left(i k\left(x_{m} u+y_{m} v\right)\right) \mid m=1,2, \ldots, M\right)
$$

is a vector-function, defined complex radiation patterns of antenna elements; $f_{0}(u, v)$ is radiation pattern of a single element; $\left(x_{m}, y_{m}\right)$ are coordinates of the $m$-th phase center antenna element; $\mathbf{A}=\left(A_{m}\right)$ is a vector of APD; $T-$ symbol of transpose; $(u, v)$ defines a direct on point view.

We introduce the matrix $\mathbf{B}=\left(B_{m, n}=1 \mid m, n=1,2, \ldots, M\right)$ of size $M \times M$ and symbol «०» which denote the Hadamar multiplication operation. In this case we can write expression

$$
F(u, v)=\mathbf{A}^{T}(\mathbf{B} \circ \mathbf{Z}) \mathbf{f}(u, v) .
$$

The formula (3) is equivalent to expression (1).

Suppose, that it is need to exclude the $n$-th element of array. So we can define $A_{n}=0$ and reset $n$-th string and row of matrix B (i.e. $B_{n, m}=B_{m, n}=0 \quad m=1,2, \ldots, \mathrm{M}$ ).

Similarly to create the passive element of AA we must to assign $A_{n}=0$ without reset elements of matrix $\mathbf{B}$ in $n$-th string and row.

Thus our model (3) allows to define three discrete states of $n$-th array element: active array element (AAE), passive array element (PAE), exclude array element (EAE).

Consider the problem of constructive synthesis of plane AA with $M$ antenna elements in follows statement. It is known:

- the coordinate grid is defined with $M$ points $\left(x_{m}, y_{m}\right)(m=1,2, \ldots, M)$ in which hypothetical $M$-element AA is situated;

- the normed resistance matrix $\mathbf{Z}$;

- vector-function $\mathbf{f}(u, v)$, defined complex radiation patterns of hypothetical antenna elements;

- the radiation pattern of AA is described expression (3).

It is required to define from the range of acceptable solutions such a vector of excitation amplitudes of the radiators $\mathbf{A}_{0}$ and the matrix $\mathbf{B}_{0}$, which provide the best quality of AA in the sense of the criterions 


$$
\begin{aligned}
& g_{1}\left(\mathbf{A}_{0}, \mathbf{B}_{0}\right)=\min _{(\mathbf{A}, \mathbf{B}) \in \mathbf{D}} g_{1,3}(\mathbf{A}, \mathbf{B}) ; \\
& g_{2,3}\left(\mathbf{A}_{0}\right)=\max _{\mathbf{A} \in \mathbf{D}} g_{2,3}(\mathbf{A}) .
\end{aligned}
$$

Here $\mathbf{D}$ is the scope of acceptable solutions;

$$
\begin{gathered}
g_{1}(\mathbf{A}, \mathbf{B})=\int_{\Omega}\left(F_{0}(u, v)-\frac{\left|\mathbf{A}^{T}(\mathbf{B} \circ \mathbf{Z}) \mathbf{f}(u, v)\right|}{\left|\mathbf{A}^{T}(\mathbf{B} \circ \mathbf{Z}) \mathbf{f}\left(u_{0}, v_{0}\right)\right|}\right)^{2} d u d v ; \\
g_{2}(\mathbf{A})=\mathbf{A}^{T} \mathbf{A}^{*}\left(\max _{m=1,2, \ldots, M}\left|A_{m}\right|^{2} \sum_{m=1}^{M} \operatorname{sign}\left|A_{m}\right|\right)^{-1} ; \\
g_{3}(\mathbf{A})=\Delta S\left(L_{x} L_{y}\right)^{-1} \sum_{m=1}^{M} \operatorname{sign}\left|A_{m}\right|
\end{gathered}
$$

are private target functions; $\left(u_{0}, v_{0}\right)$ - direct of radiation pattern maximum; $\Delta S$ - square of single antenna element;

$$
\begin{aligned}
& L_{x}=\max _{m=1,2, \ldots, M} \operatorname{sign}\left(\left|A_{m}\right| x_{m}\right)-\min _{m=1,2, \ldots, M} \operatorname{sign}\left(\left|A_{m}\right| x_{m}\right) ; \\
& L_{y}=\max _{m=1,2, \ldots, M} \operatorname{sign}\left(\left|A_{m}\right| y_{m}\right)-\min _{m=1,2, \ldots, M} \operatorname{sign}\left(\left|A_{m}\right| y_{m}\right)
\end{aligned}
$$

are sizes of AA along coordinate axes without account of exclude antenna elements;

$$
\operatorname{sign} x=\left\{\begin{array}{cc}
1, & x>0 \\
0, & x=0 \\
-1, & x<0
\end{array}\right.
$$

Consider the NN which consist of one classifying part and two approximating parts (fig. 1). Input signals we define as normed coordinates of hypothetical antenna elements of AA:

$$
\xi_{1}=x\left(\max _{m=1,2, \ldots, M}\left(x_{m}\right)\right)^{-1} ; \quad \xi_{2}=y\left(\max _{m=1,2, \ldots, M}\left(y_{m}\right)\right)^{-1} .
$$

Each part of NN has fully conected structure. Classifying part has three output for each discrete state of element (AAE, PAE, EAE). Approximating parts have one output for each one. The output signal of the first approximating part correspond to amplitude of element $|A(\xi)|$, and the output signal of the second part - to the phase $\arg A(\xi) / \pi$. The attachment of element to the one from three classes we define by the number of maximum output signal in classifying part.

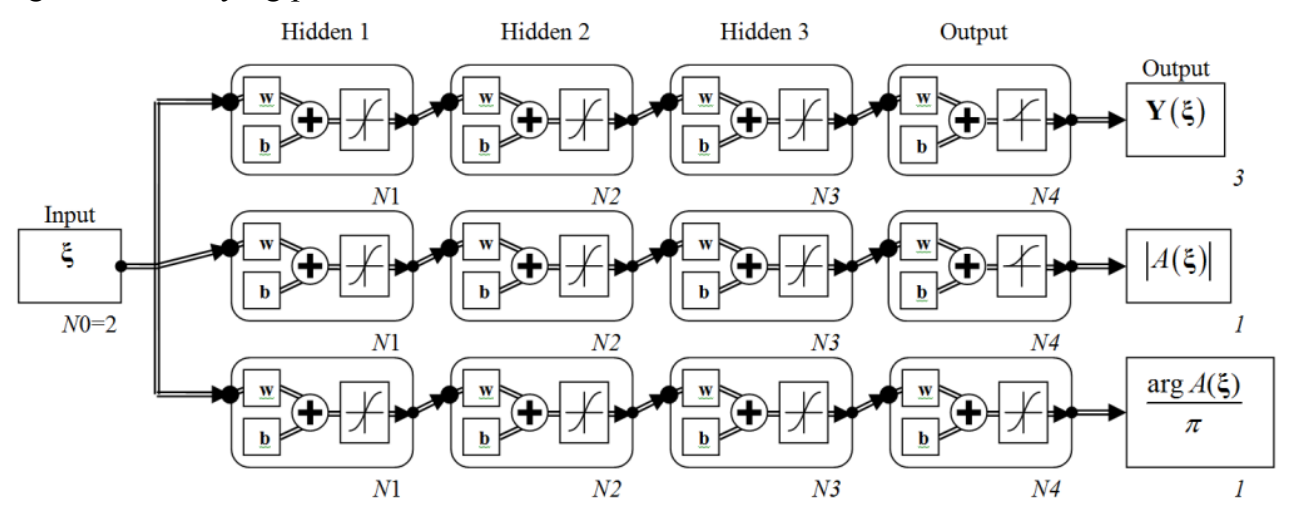

Fig. 1. The structure of NN. 
All parts of $\mathrm{NN}$ have three hidden layers. We selected $a_{1}(x)=\operatorname{tgh} x$ as activation function in hidden layers. In output leyer of the classifying part and first approximating part we used sigmoid activate function $a_{2}(x)=(1+\exp (-x))^{-1}$.

The mathematical model of NN with selected structures can be presented in the sight

$$
\begin{gathered}
\mathbf{Y}(\xi)=a_{1}\left(\mathbf{w}_{4}^{\langle 1\rangle} a_{1}\left(\mathbf{w}_{3}^{\langle 1\rangle} a_{1}\left(\mathbf{w}_{2}^{\langle 1\rangle} a_{1}\left(\mathbf{w}_{1}^{\langle 1\rangle} \xi+\mathbf{b}_{1}^{\langle 1\rangle}\right)+\mathbf{b}_{2}^{\langle 1\rangle}\right)+\mathbf{b}_{3}^{\langle 1\rangle}\right)+\mathbf{b}_{4}^{\langle 1\rangle}\right) ; \\
|A(\xi)|=a_{2}\left(\mathbf{w}_{4}^{\langle 2\rangle} a_{1}\left(\mathbf{w}_{3}^{\langle 2\rangle} a_{1}\left(\mathbf{w}_{2}^{\langle 2\rangle} a_{1}\left(\mathbf{w}_{1}^{\langle 2\rangle} \boldsymbol{\xi}+\mathbf{b}_{1}^{\langle 2\rangle}\right)+\mathbf{b}_{2}^{\langle 2\rangle}\right)+\mathbf{b}_{3}^{\langle 2\rangle}\right)+\mathbf{b}_{4}^{\langle 2\rangle}\right) ; \\
\pi^{-1} \arg A(\xi)=a_{1}\left(\mathbf{w}_{4}^{\langle 3\rangle} a_{1}\left(\mathbf{w}_{3}^{\langle 3\rangle} a_{1}\left(\mathbf{w}_{2}^{\langle 3\rangle} a_{1}\left(\mathbf{w}_{1}^{\langle 3\rangle} \xi+\mathbf{b}_{1}^{\langle 3\rangle}\right)+\mathbf{b}_{2}^{\langle 3\rangle}\right)+\mathbf{b}_{3}^{\langle 3\rangle}\right)+\mathbf{b}_{4}^{\langle 3\rangle}\right),
\end{gathered}
$$

where $\mathbf{w}_{j}^{\langle i\rangle}$ and $\mathbf{b}_{j}^{\langle i\rangle}$ - the matrices of communications coefficients and the offsets vectors $i$-th part and $j$-th layer of NN.

In fact, APD of the AA can be expressed through output signal of NN with help of the expression:

$$
A_{m}=\left\{\begin{array}{cl}
\left|A\left(\xi_{m}\right)\right| \exp \left(i \arg A\left(\xi_{m}\right)\right), & Y_{1}\left(\xi_{m}\right)=\max \mathbf{Y}\left(\xi_{m}\right) \\
0, & Y_{1}\left(\xi_{m}\right) \neq \max \mathbf{Y}\left(\xi_{m}\right) .
\end{array}\right.
$$

Here $\xi_{m}$ are defined with (12) for each coordinate pair $\left(x_{m}, y_{m}\right)$.

The elements of binary matrix $\mathbf{B}$ are calculated by the formula

$$
B_{m, n}=B_{n, m}\left\{\begin{array}{ll}
1, & Y_{3}\left(\xi_{m}\right) \neq \max \mathbf{Y}\left(\xi_{m}\right) ; \\
0, & Y_{3}\left(\xi_{m}\right)=\max \mathbf{Y}\left(\xi_{m}\right) ;
\end{array} n=1,2, \ldots, M\right.
$$

The arbitrary sets of coefficients $\mathbf{w}_{j}^{\langle i\rangle}$ and $\mathbf{b}_{j}^{\langle i\rangle}$ belong to forming $\mathbf{A}, \mathbf{B} \in \mathbf{D}$. To the forming $\mathbf{A}_{0}, \mathbf{B}_{0}$ it is need to train our NN.

\section{Learning algorithm}

The process of NN training devide on two stage. The first stage consist of separate learning each parts of NN. Second stage need for common training.

\subsection{The first stage learning algorithm}

The first stage based on follow ideas.

In first, all known AA with active and passive elements has one structure: active elements placed in the center of aperture, passive elements placed along perimeter of active part of AA. So preliminary training of classifying part we can perform on criterion of the minimum standard deviation:

$$
\begin{aligned}
& g_{4}\left(\mathbf{w}_{j}^{\langle 1\rangle}, \mathbf{b}_{j}^{\langle 1\rangle}\right)=\int_{-1}^{1} \int_{-1}^{1}\left(\mathbf{Y}\left(\xi_{1}, \xi_{2}, \mathbf{w}_{j}^{\langle 1\rangle}, \mathbf{b}_{j}^{\langle 1\rangle}\right)-\hat{\mathbf{Y}}\left(\xi_{1}, \xi_{2}\right)\right)^{T} \times \\
& \times\left(\mathbf{Y}\left(\xi_{1}, \xi_{2}, \mathbf{w}_{j}^{\langle 1\rangle}, \mathbf{b}_{j}^{\langle 1\rangle}\right)-\hat{\mathbf{Y}}\left(\xi_{1}, \xi_{2}\right)\right) d u_{1} d u_{2},
\end{aligned}
$$

where

$$
\hat{\mathbf{Y}}(\xi)=\hat{\mathbf{Y}}\left(\xi_{1}, \xi_{2}\right)=\left\{\begin{array}{lc}
(1,0,0)^{T}, & \xi^{T} \xi<U_{1} \\
(0,1,0)^{T}, & U_{1} \leq \xi^{T} \xi<U_{2} \\
(0,0,1)^{T}, & U_{2} \leq \xi^{T} \xi \leq 1
\end{array}\right.
$$


$U_{1}<U_{2}<1$ are parameters which deterrmine sizes of regions with AAE and EAE.

In second, for known constructive synthesis problems set the radiation pattern is symmetrical function. It allows to find the APD in the hypothetical aperture of AA which consist of only AAE. So preliminary training of first approximating part can be fullfil on minimum criterion of follow target function

where

$$
g_{5}\left(\mathbf{w}_{j}^{\langle 2\rangle}, \mathbf{b}_{j}^{\langle 2\rangle}\right)=\sum_{m=1}^{M}\left(A\left(\boldsymbol{\xi}_{m}, \mathbf{w}_{j}^{\langle 2\rangle}, \mathbf{b}_{j}^{\langle 2\rangle}\right)-\hat{A}_{m}\right)^{2},
$$

$$
\begin{gathered}
\hat{\mathbf{A}}=\left(\hat{A}_{m} \mid m=1,2, \ldots, M\right)=\boldsymbol{\eta} \mathbf{S}^{-1} ; \\
\boldsymbol{\eta}=\int_{u^{2}+v^{2} \leq 1} F_{0}(u, v) \mathbf{f}^{H}(u, v) d u d v ; \mathbf{S}=\int_{u^{2}+v^{2} \leq 1} \mathbf{f}(u, v) \mathbf{f}^{H}(u, v) d u d v ;
\end{gathered}
$$

$H$ denote the Hermitian conjugation.

The second approximating part of $\mathrm{NN}$ training for forming inphase distribution in the aperture. To decision this problem could be used target function

$$
g_{6}\left(\mathbf{w}_{j}^{\langle 3\rangle}, \mathbf{b}_{j}^{\langle 3\rangle}\right)=\frac{1}{\pi^{2}} \sum_{m=1}^{M}\left(\arg A\left(\mathbf{u}_{m}, \mathbf{w}_{j}^{\langle 3\rangle}, \mathbf{b}_{j}^{\langle 3\rangle}\right)\right)^{2} .
$$

The target functions (18), (20) и (23) acceptably use with known error back propagation method [5].

\subsection{The second stage of learning algorithm}

In the second stage, criteria (4) and (5) must be used to train the NN. For solving problems of multicriteria optimization typically use the procedure of scalarization vector criterion and perform the tuning of a NN with algorithms for the directed search.

We choose a genetic algorithm to implement a directional search procedure. The genetic algorithm is that they form a sample of admissible solutions (the population) from which a subset of the best solutions is selected (the parent set). Selected solutions are used to form a new population. The main problem of this approach is that with an increase in the dimension of the problem, the criterion for selecting the elements of the parent set may lose sensitivity. This will lead to stagnation of the problem solving process. In this regard, the use of a genetic algorithm to solve the problem in question involves solving the problem of high dimensionality of the neural network learning problem.

In [4], a set of constant coefficients was used to scalarize a vector criterion, the choice of which is difficult to substantiate. In this paper it is proposed to use a lexicographic approach to the scalarization of a multi-criteria task. It consists in the fact that private criteria are used in a given order in order to finally get the single solution. In this case private objective functions were proposed to be used in order. The first particular objective functions take into account the peculiarities of the placement of active and passive elements in the aperture. The third partial objective function takes into account the requirements for the form of radiation pattern and allows to obtain a single solution. As a result, the criteria (5) in the learning process were replaced by inequalities

$$
g_{2}\left(\mathbf{A} \in \mathbf{D}_{2}\right) \geq \overline{g_{2}\left(\mathbf{A} \in \mathbf{D}_{1} \subset \mathbf{D}\right)} ; \quad g_{3}\left(\mathbf{A} \in \mathbf{D}_{3}\right) \geq \overline{g_{3}\left(\mathbf{A} \in \mathbf{D}_{2} \subset \mathbf{D}_{1}\right)},
$$

where $\mathbf{D}_{1}$ is the initial subset of admissible solutions from the set $\mathbf{D} ; \mathbf{D}_{2}$ is a subset of admissible solutions selected with first inequalities (24) from the subset $\mathbf{D}_{1}$; $\overline{g_{2}\left(\mathbf{A} \in \mathbf{D}_{1} \subset \mathbf{D}\right)}$ and $\overline{g_{3}\left(\mathbf{A} \in \mathbf{D}_{2} \subset \mathbf{D}_{1}\right)}$ are average values of target functions on the subsets $\mathbf{D}_{1}$ and $\mathbf{D}_{2} ; \mathbf{D}_{3}$ is a subset of solutions selected with second inequalities in (24). 
To reduce the size of the learning task, the learning algorithm was proposed to be divided into a series of successive learning epochs, in each of which the number of NN coefficients trained according to the genetic algorithm was limited. This approach was considered in the monograph [6].

Thus, the proposed method of constructive synthesis of AA, taking into account the mutual influence of elements, is reduced to using the neural network approach and performing the following operations:

- formation of the NN structure corresponding to fig. 1;

- preliminary independent infusion of parts of $\mathrm{NN}$;

- training the NN of fragments of a common NN based on the analysis of the values of target functions in accordance with the established lexicographic order of criterias of the form (24), as well as the minimum of the target function (6).

The mutual influence of elements in the composition of the AA takes into account the mathematical model of the AA of the form (3) with the division of AE into three classes: AAE, PAE and EAE.

The novelty of the proposed method of constructive synthesis of AA consists in using the corresponding NN structure and the chosen lexicographical order of application of criterias reflecting the requirements for sensitivity or radiated power of the AA, the compactness of the placement of active elements, as well as the quality of formation of a preassigned radiation pattern.

\section{Efficiency evaluation of the proposed method}

As example, we consider the solution of the problem of constructive synthesis of AA of thin half-wave $x$-polarized vibrators located with horizontal and vertical steps $d_{x}=0,52 \lambda$ and $d_{y}=0,48 \lambda$. We assume that the synthesized AP should fit into a rectangular aperture and consist of $40 \times 40$ antenna elements.

For the $x$-component of the radiation pattern of a single antenna element, we used the following expression

$$
f_{0}(u, v)=\cos (k l u)\left(1-u^{2}\right)^{-1},
$$

where $l=0,25 \lambda$ is a vibrator shoulder length.

To determine the matrix of mutual resistances $\mathbf{Z}$, we used the formulas for calculating the electromagnetic field in the near zone of the vibrator, given in [10].

The preassigned radiation pattern was defined using the expression of the form

$$
F_{0}(u, v)=\left\{\begin{array}{cc}
\cos \left(\frac{\pi}{4} \frac{\sqrt{u^{2}+v^{2}}}{\sin \left(2,5^{\circ}\right)}\right), & \arcsin \sqrt{u^{2}+v^{2}} \leq 4^{o} ; \\
0,01, & 4^{o}<\arcsin \sqrt{u^{2}+v^{2}} \leq 8^{o} ; \\
0, & \arcsin \sqrt{u^{2}+v^{2}}>8^{\circ} .
\end{array}\right.
$$

It is required to find a solution of the problem of constructive synthesis, which is the best in the sense of criterias (24) and (4).

To solve the problem, we used the NN shown in Fig. 1. The number of neurons in the hidden layers is chosen equal to $N 1=100, N 2=200, N 3=48$.

Preliminary training of the first part of the NN requires the formation of vector-function $\hat{\mathbf{Y}}(\xi)$ values in accordance with the expression (19). The $U_{1}=0,6$ and $U_{2}=0,8$ 
parameter values were selected for this purpose. The number of iterations for pre-training was 500 .

Training of the entire NN was carried out on 30 lists of coefficients. Each list consisted of 300 numbers of coefficients in each part of the NN (i.e. the total number of simultaneously trained coefficients of the $\mathrm{NN}$ was 900). The list contained the offset coefficients of the output layer and the last hidden layer. The remaining number of learners coefficients were chosen using the random number generator.

To determine the coefficients of the neural network from the generated list, a genetic algorithm was used. The population length of the coefficients (subset power $\mathbf{D}_{1}$ ) was chosen to be 100 . The length of the parent set was chosen to be 5 . When forming a new population, the parameters of the genetic algorithm were used: $p_{r}=0,2-$ the probability of crossing (the probability that the chromosome will contain the gene of not the best parent) and $p_{m}=0,9-$ the probability of mutation of the current gene. Each population included elements of the parent set to ensure the continuity of the quality of the solution from one generation to another.

The number of generations in the implementation of the genetic algorithm was variable and varied from 5 to 15 as the number of iterations corresponding to the number of the list of coefficients. Fig. 2 shows a curve that reflects the quality of the radiation pattern formation during neural network training. It can be seen that after the 3 rd iteration, the quality of training has decreased significantly, but when the remaining 30 iterations were observed monotonous improvement in the accuracy of the approximation of the generated radiation pattern to a preassigned radiation pattern.

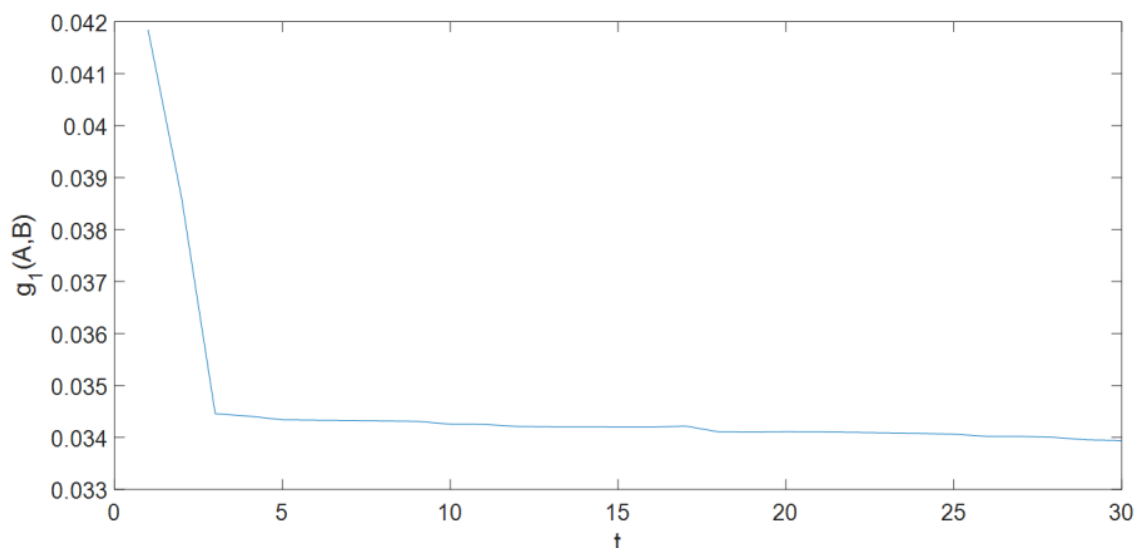

Fig. 2. Convergence of the solution.

Fig. 3 shows the boundary of the opening area, inside which 828 AAE of the synthesized AA are located. In the same figure, the positions of 16 PAE are marked with dots.

Fig. 4 and 5 demonstrate the amplitude and phase distributions in the AA aperture.

Fig. 6 shows the volume radiation pattern of the AA.

The analysis of the obtained results shows that the proposed method allows to obtain solutions to the problems of multi-criteria constructive synthesis of AA taking into account the mutual relationship between the elements. In this case, the resulting solution allows to evaluate not only the coordinates of the AAE, but also to form the configuration of the aperture with PAE. 


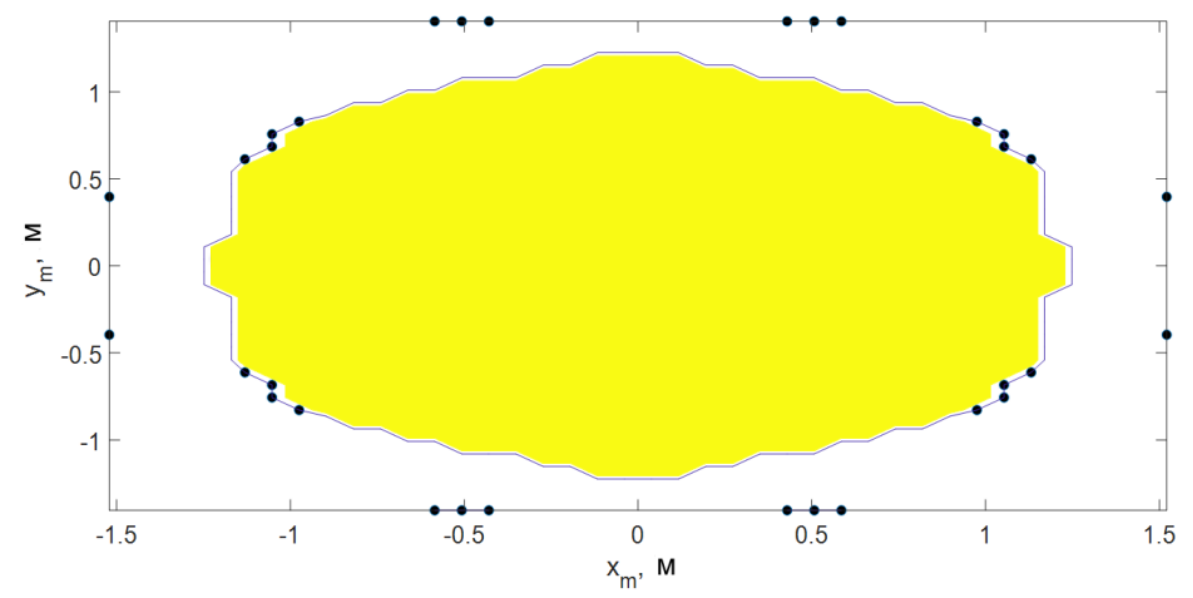

Fig. 3. The form of the border of the active part of the aperture of $828 \mathrm{AAE}$ and the provision of 16 PAE.

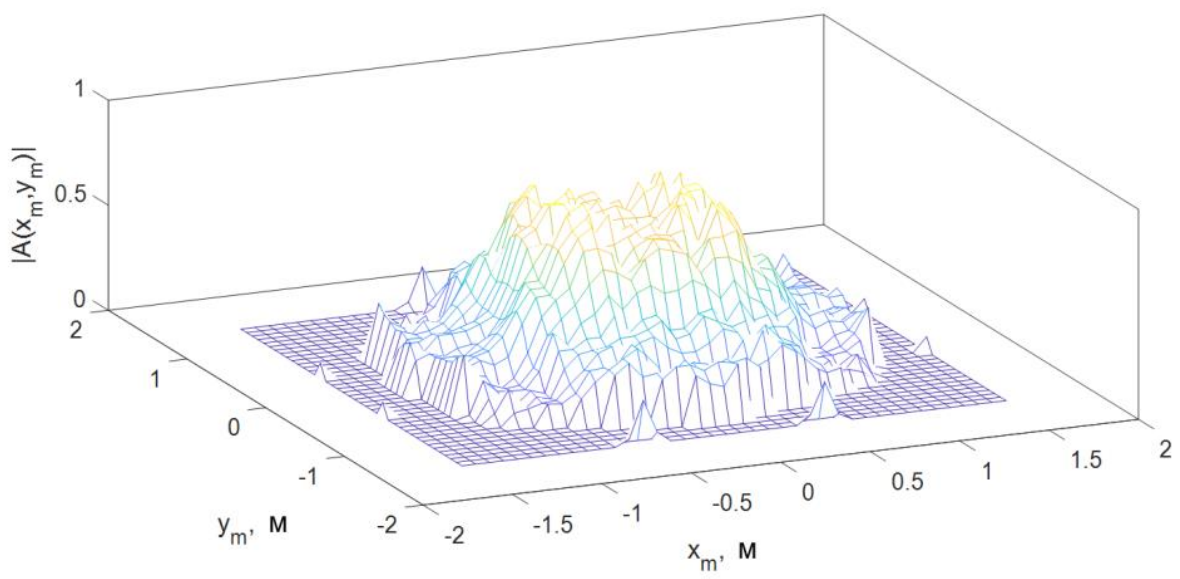

Fig. 4. The amplitude distribution in AA aperture.

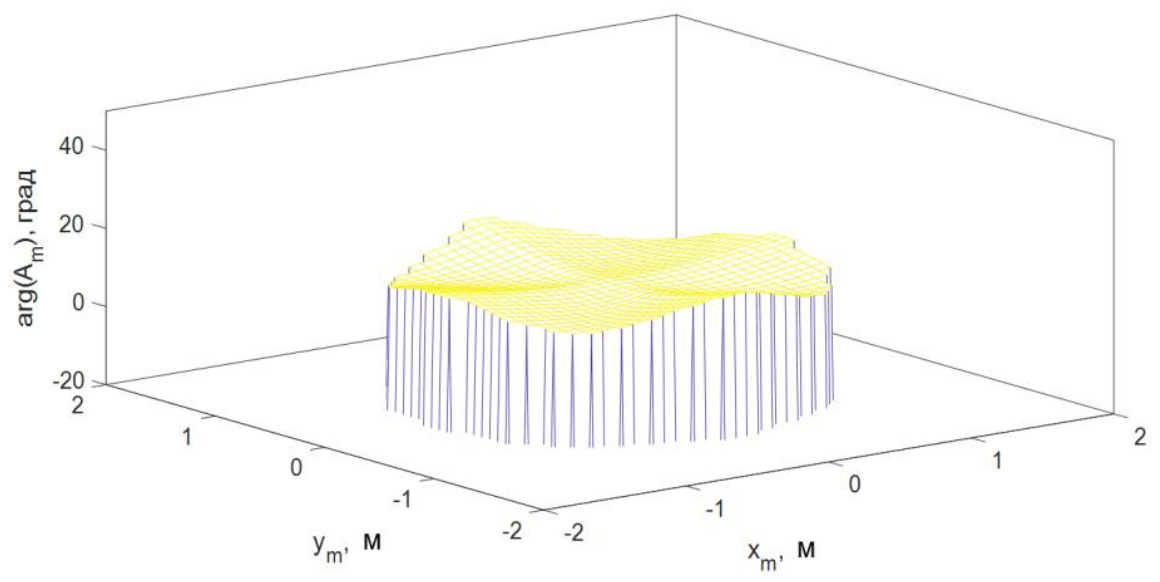

Fig. 5. The phase distribution in AA aperture. 


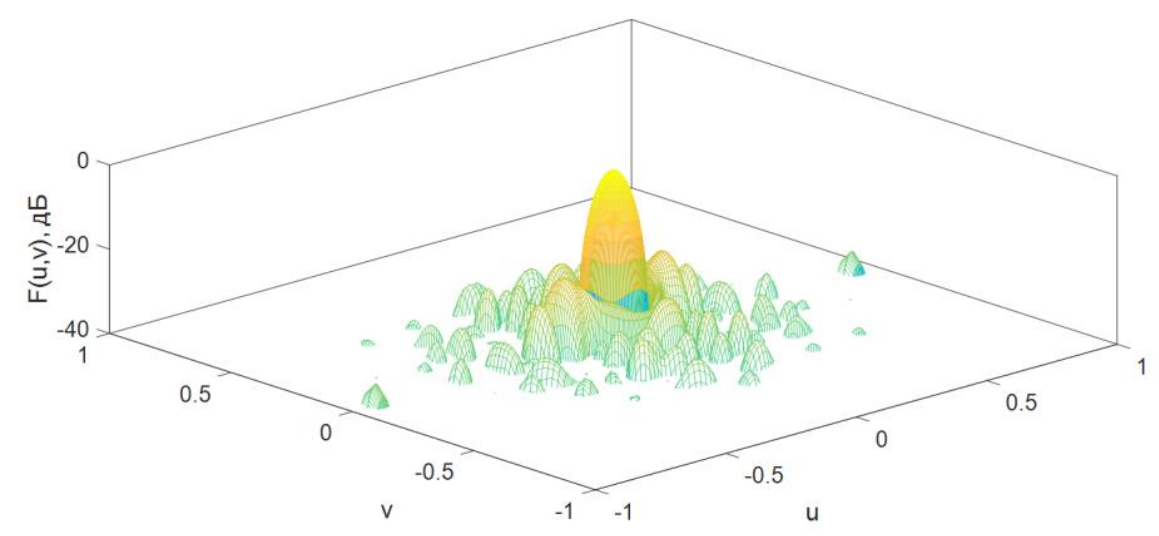

Fig. 6. 3D radiation pattern.

\section{Conclusions}

1. The proposed method of constructive synthesis of AA differs from the known taking into account the mutual influence of antenna elements and the use of a neural network approach to select the coordinates of AAE and PAE.

2. The results confirm the efficiency of the proposed method of constructive synthesis of $\mathrm{AA}$ and the convergence of the algorithm of $\mathrm{NN}$ training.

\section{References}

1. E.G. Zelkin, V.G. Sokolov Methods of synthesis of antennas: phased antenna arrays and antennas with continuous aperture 296 (1980)

2. E.G. Zelkin, V.F. Kravchenko, V.I. Gusevskiy Constructive approximation methods in antenna theory 512 (2005)

3. N.N. Voitovich, B.Z. Kacenelenbaum, N.N. Korshunova and other Electrodynamics of antennas with half-transparent surfaces: Methods of constructive synthesis 176 (1989)

4. A.A. Bezuglov, S.E. Mishchenko, V.V. Shatskiy Proceedings of the 3rd all-Russian Microwave conference 100 (2015)

5. A.A. Bezuglov, S.E. Mishchenko, V.V. Shatskiy Antennas, 3 (2015)

6. S.E. Mishchenko, V.V. Shatskiy, D.Yu. Eliseev and other Proceedings of RLINC, 369 (2019)

7. A.V. Nazarov, A.I. Loskutov Neural network prediction and optimization algorithms, $384(2003)$

8. L.D. Bahrah, S.D. Kremenetskiy Synthesis of radiating systems. Theory and methods of calculation, 232 (1974)

9. A.P. Karpenko Modern search engine optimization algorithms. Nature-inspired algorithms, 446 (2014)

10. G.T. Markov, D.M. Sazonov Antennas, 528 (1975) 